\title{
ADRIAMYCIN-INDUCED FETAL HYDRONEPHROSIS
}

\author{
ANDERSON GONÇALVES, WILLY G. FRANÇA, SUZANA G. MORAES, LUIS A.V. PEREIRA, \\ LOURENÇO SBRAGIA
}

\author{
Institute of Biology and School of Medicine, State University of Campinas, UNICAMP, Campinas,
} São Paulo, Brazil

\begin{abstract}
Introduction: At the end of pregnancy, the amniotic fluid (AF) depends basically on renal function, corresponding to fetal urine. Changes in $\mathrm{AF}$, especially oligohydramnios, are reported in association with fetal hydronephrosis $(\mathrm{FH})$. The experimental model using adriamycin in pregnant female rats has a teratogenic effect and has been classically employed to study esophageal atresia. Nevertheless, adriamycin promotes FH with high frequency as well. In the present study, using this animal model, we tried to identify the incidence and microscopic changes of FH, as well as its correlation with $\mathrm{AF}$ weight.

Materials and Methods: Eight Spreague-Dawley pregnant female rats received adriamycin $2.2 \mathrm{mg} / \mathrm{kg}$ on the 8th and 9th gestational days (considering term gestation $=22$ days). Those fetuses that received adriamycin (Adriamycin Group) were compared with fetuses from 2 female rats (Control Group), which received $0.9 \%$ saline solution. On the 21.5 gestational day, the fetuses were collected by cesarean incision, sacrificed, and examined for macro and microscopic changes in kidneys and ureters. Fetuses with bilateral hydronephrosis formed the Hydronephrosis Group. AF weight was determined as well.

Results: Hydronephrosis occurred in 70 (95\%) of the 74 fetuses in the adriamycin group against none of the 21 fetuses from the control group. The amniotic fluid weight was increased in the adriamycin group in relation to the control group $(\mathrm{p}<0.001)$. The histomorphometric study revealed dilation of the renal pelvis and reduction of renal parenchyma in the hydronephrosis group in relation to the control group. Severe cortical atrophy, cortical tubular atrophy and medullar atrophy were observed in the hydronephrosis group.

Conclusions: Slight renal lesions were in agreement with changes in AF weight, since they suggest that there was production of urine with the maintenance of AF.
\end{abstract}

Key words: rats; amniotic fluid; fetus; adriamycin; hydronephrosis

Int Braz J Urol. 2004; 30: 508-13

\section{INTRODUCTION}

The amniotic fluid (AF) at the end of pregnancy depends basically on renal function, corresponding to the fetal urine (1). Changes in AF concerning volume, osmolarity and solute partition are reported in association with fetal hydronephrosis $(\mathrm{FH})$ (2-4).
FH is characterized by prenatal dilation of the renal pelvis. Generally, it is associated with decreased urinary flow, structural changes in renal parenchyma and impairment of renal function $(5,6)$. The clinical manifestation of $\mathrm{FH}$ is variable, presenting an unfavorable outcome when it is bilateral or is associated with decrease in AF (oligohydramnios) $(2,7,8)$. Moreover, the prenatal assessment of AF has 
a predictive value on the prognosis of the newborn $(7,8)$.

The experimental model using adriamycin in pregnant female rats has a teratogenic effect and has been classically employed to study esophageal atresia. However, adriamycin promotes other fetal morphologic changes, with FH being the urinary anomaly that occurs with higher frequency (9-11). Much has been described about gastrointestinal changes in the adriamycin model (12), however, little has been studied about renal changes and their correlation with AF.

In the present study, we tried to identify the feasibility of using adriamycin for the microscopic study of $\mathrm{FH}$, aiming to correlate renal microscopic changes with $\mathrm{AF}$ weight ( $\mathrm{AFw}$ ).

\section{MATERIALS AND METHODS}

Eight Spreague-Dawley pregnant female rats, weighting between 250 and $300 \mathrm{~g}$, received intra-peritoneal adriamycin $2.2 \mathrm{mg} / \mathrm{kg}$ on the 8 th and 9 th gestational days (term $=22$ days). The fetuses that received adriamycin (Adriamycin Group) were compared with fetuses from 2 female rats (Control Group) that had received $0.9 \%$ saline solution on the same gestational days.

On the day 21.5 of pregnancy, the rats underwent a cesarean incision and the amniotic sac was integrally extracted and weighted ( $\mathrm{ASw}$ ) in a precision balance. The amniotic sac was then excised and the fetus $(\mathrm{Fw})$, the placenta and the amniochorionic membranes (PMw) were weighted separately. The $\mathrm{AFw}$ was obtained through the formula: $\mathrm{AFw}=\mathrm{ASw}$ - $(\mathrm{FW}+\mathrm{PMw})$ in grams.

Then the fetuses were collected, sacrificed and examined for macroscopic changes in kidneys and ureters, bladder changes, and presence of proximal digestive atresias. Fetuses from the adriamycin group with bilateral hydronephrosis formed the Hydronephrosis Group.

The abdominal region with the retroperitoneal cavity of fetuses, containing kidneys and ureters, was fixed in formaldehyde $4 \%$ and included in paraffin. Semi-serial coronal histological sections were obtained, measuring $5 \mu \mathrm{m}$, equidistant in $20 \mu \mathrm{m}$ between the anterior and posterior renal limits. Sections were stained with hematoxylin and eosin.

\section{Histomorphometric Study and Qualitative Microscopic Analysis}

The fetal kidneys from the hydronephrosis group and the control group were compared under light microscopy. Images obtained by microscopy were transmitted to the computer via digital camera. Subsequently, they were dimensioned using ImagePro Plus 4.1 software (Media Cybernetics 1999), which allows the gauging of linear measures and area after manually defining 2 points and the perimeter, respectively. The micrometric scale was previously defined by a calibration file, according to the microscope's objective lens.

With a X20 magnification, the 3 consecutive sections of each left kidney were determined, where the diameters of the ureteropelvic junction were largest. In these sections, the diameter of the ureteropelvic junction, the mean parenchymal thickness, the area of the renal pelvis, and the area of renal parenchyma were measured, and the relationships between parenchyma and pelvis were established (Figure-1).

The histological changes of pelvic epithelium, proximal ureter and kidney (epithelial, mesenchymal, epithelial-mesenchymal, obstructive and inflammatory) were qualitatively analyzed according to what is described in the literature for the model of obstructive hydronephrosis $(13,14)$.

\section{Statistical Analysis}

The statistical analysis of weights, with comparisons between the adriamycin and control groups, and the histomorphometric measures, with comparisons between the hydronephrosis and control groups, was performed through Mann-Whitney non-parametric test, considering the difference as significant for $\mathrm{p}<0.05$ and highly significant for $\mathrm{p}<0.001$.

\section{RESULTS}

Pregnancy was confirmed in 10 female rats, with 8 forming the adriamycin group and 2 the control group. A total of 81 fetuses were obtained in the 


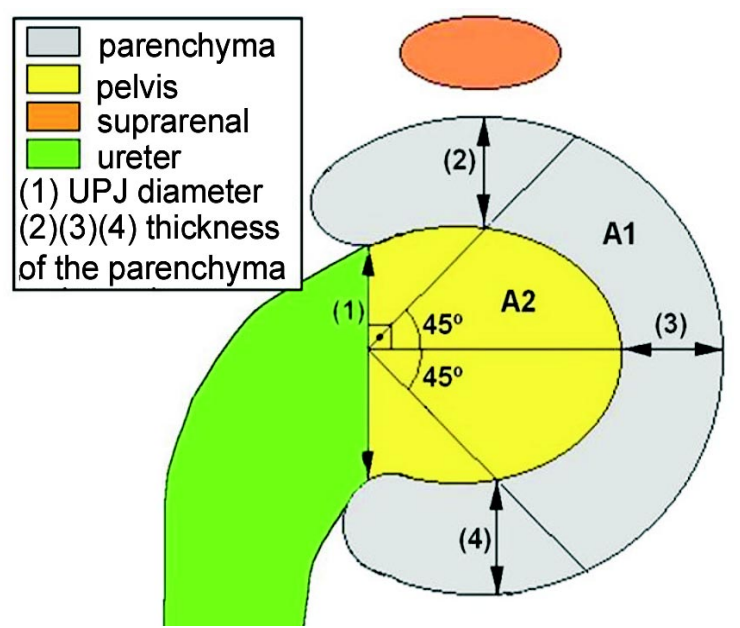

Figure 1 - Scheme used for performing the histomorphometric examination. 2-headed arrows indicate linear measurements. $A 1=$ area of renal parenchyma and $A 2=$ area of renal pelvis.

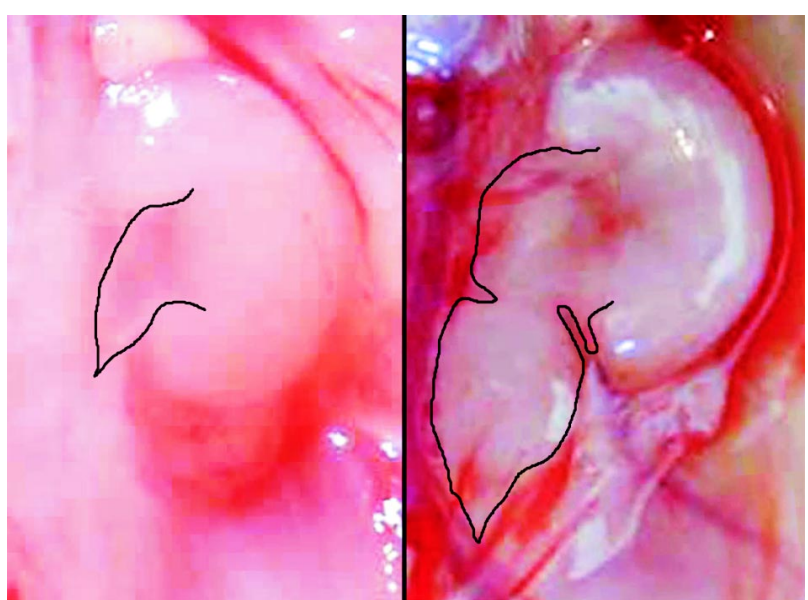

Figure 2 - Macroscopic comparison between a normal kidney from the control group (left) with hydronephrotic kidney from the adriamycin group (right). The ureters are delimited, showing dilation in the adriamycin group.
Table 1 - Frequency of malformations in the adriamycin group.

\begin{tabular}{lc}
\hline Malformation & Frequency (\%) \\
\hline Hydronephrosis & $70(95)$ \\
- Bilateral & $64(87)$ \\
- Unilateral & $6(8)$ \\
Duodenal Atresia & $68(92)$ \\
Esophageal Atresia & $67(91)$ \\
Bladder Hypoplasia & $62(84)$ \\
\hline
\end{tabular}

adriamycin group, of which 74 (91\%) were alive and 7 (9\%) dead (4 were reabsorbed and 3 were hydropic). In the control group, 21 fetuses were obtained, all alive.

In the adriamycin group, hydronephrosis (Figure-2) was observed in 70 out of 74 fetuses (95\%), in addition to other malformations. In the 21 fetuses from the control group, the malformations under study were not observed (Table-1).

$\mathrm{ASw}, \mathrm{Fw}$ and $\mathrm{PMw}$ were decreased and $\mathrm{AFw}$ was increased in the adriamycin group in relation to the control group ( $\mathrm{p}<0.001)$ (Table-2).

The histomorphometric study revealed pelvic dilation and reduction of renal parenchyma in the hydronephrosis group when compared with the control group ( $\mathrm{p}<0.001)$ (Table-3).

The qualitative microscopic analysis of the kidneys in both groups demonstrated the capacity of distinguishing between medullar and cortical in a lower magnification (X20) and the existence of nephrogenic zone, composed by immature glomeruli (Figure-3). In the hydronephrosis group, we observed se-

Table 2 - Comparison of weights. Mean, standard deviation and interval.

\begin{tabular}{llc}
\hline Weights & Adriamycin Group & Control Group \\
\hline ASw $(\mathrm{g})$ & $5.23^{*}( \pm 0.56) ; 0.38-6,51$ & $5.96( \pm 0.27) ; 5.27-6.63$ \\
Fw $(\mathrm{g})$ & $3.39^{*}( \pm 0.43) ; 2.12-4.36$ & $4.27( \pm 0.18) ; 3.88-4.62$ \\
$\mathrm{PMw}(\mathrm{g})$ & $0.95^{*}( \pm 0.16) ; 0.35-1.62$ & $1.11( \pm 0.14) ; 0.67-1.50$ \\
$\mathrm{AFw}(\mathrm{g})$ & $0.90^{*}( \pm 0.33) ; 0.08-2.34$ & $0.58( \pm 0.18) ; 0.19-0.97$ \\
\hline
\end{tabular}

${ }^{*} p<0.001 ; A S w=$ weight of amniotic sac; $F w=$ fetal weight PMw = weight of placenta and amniotic membranes; AFw = weight of amniotic fluid. 
Table 3 - Comparison of histomorphometric measures. Mean, standard deviation and interval.

\begin{tabular}{llc}
\hline Relationship & Hydronephrosis Group & Control Group \\
\hline $\begin{array}{l}\text { Parenchymal Thickness } \\
\quad \text { X UPJ Diameter } \\
\begin{array}{l}\text { Parenchymal Area } x \\
\text { Pelvic Area }\end{array}\end{array}$ & $0.68^{*}( \pm 0.25) ; 0.37-1.52$ & $1.36( \pm 0.30) ; 0.81-2.10$ \\
\hline
\end{tabular}

${ }^{*} p<0.001 ;$ UPJ $=$ ureteropelvic junction

vere cortical atrophy and tubular cortical atrophy; as well as moderate caliceal dilation, moderate to severe cystic tubular change and slight mesangial hyperplasia, whereas in the control group such changes were not observed (Figure-4).

The search for microscopic changes in pelvis and ureter revealed severe pelvic dilation with flattening of the epithelial cells, and dilation and tortuosity of the proximal ureter in the hydronephrosis group. On the other hand, no changes were observed in the ureteral epithelium, which was similar to the control group.

\section{COMMENTS}

Adriamycin acts on the S-phase of the cell cycle, inhibiting topoisomerase II and consequently

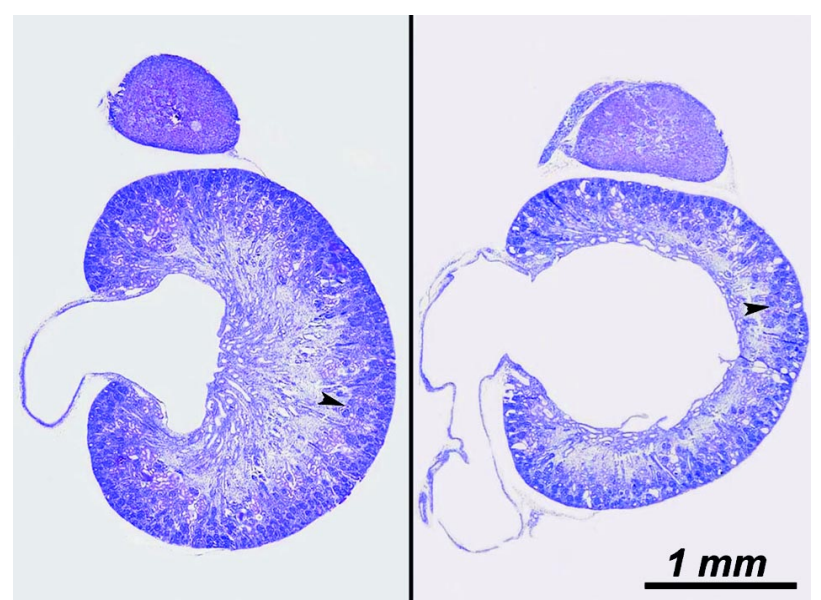

Figure 3 - Microscopic comparison between a normal kidney from the control group (left) with hydronephrotic kidney from the hydronephrosis group (right). The cortical-medullar limit is shown (arrowheads). Note the atrophy in the hydronephrosis group, (HE, X20).

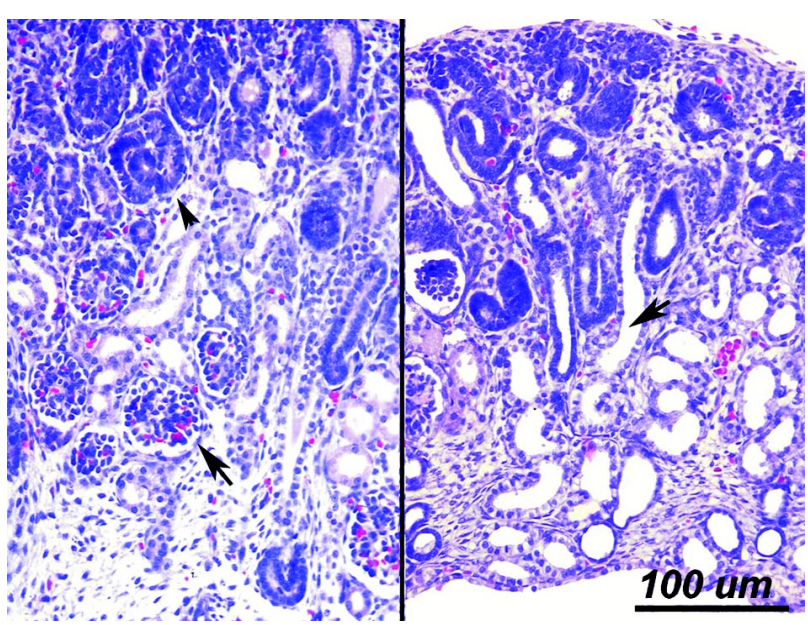

Figure 4 - Microscopic comparison between a normal kidney from the control group (left) with hydronephrotic kidney from the hydronephrosis group (right). Note mature (arrows) and immature (arrowheads) glomeruli in the control group. In the hydronephrosis group, we can see medullar and cortical atrophy and cystic tubular dilation (arrow), (HE, X200).

DNA synthesis (15). This inhibition induces cell apoptosis, and is the potential molecular cause of the malformations detected, deriving mostly from a failure in the embryologic development of mesoderma (16).

The decrease in ASw, Fw and PMw in the adriamycin group in relation to the control group is due to the drug's deleterious effects, either by its primary action, or by the repercussions from the malformations it induces $(9,10)$.

The frequency of detected malformations was similar to those found by other authors who used adriamycin $(9-11,17)$.

Fetuses with serious renal defects leading to intra-uterine urinary retention evolve with oligohy- 
dramnios, due to the relation between the production of fetal urine and a proper amount of AF $(3,7)$. Despite bilateral $\mathrm{FH}$ occurring in $87 \%$ of fetuses in the adriamycin group, there was an increase in $\mathrm{AFw}$ in relation to the control group $(\mathrm{p}<0.0001)$. However, a bilateral FH occurred in association with $100 \%$ of digestive atresias. Such atresias, especially proximal ones, impair the deglutition of AF, as well as its absorption in the gastrointestinal tract, and may lead to polyhydramnios (18).

The association between these anomalies, intestinal atresias and $\mathrm{FH}$, which have a contrary effect on AFw, and other potential causes of oligohydramnios can make results controversial. However, the present study showed that the ability of forming urine, and consequently AF by the fetal kidney was preserved, since there was no decrease in the AFw. Hence, it is not important to say that AFw increased, but that it did not decrease, since little AF would indicate low production of fetal urine.

Merei et al. (2001) studied urinary malformations in the adriamycin model and concluded that the ureters would present a blind end, producing hydronephrotic fetal kidneys and altering the normal development of urinary bladder (11). In order to explain the increase in AF that occurs concomitantly in blind-ending ureters, extra-renal compensatory mechanisms would be required.

Liu \& Hutson (2000), while studying urogenital malformations in the same model, concluded that the ureters might communicate with the urethra in male fetuses, and with the urogenital sinus in female fetuses, through an uretero-ureteral or uretero-urogenital fistula, respectively (19). Thus, urinary flow would occur, which would allow for a proper maintenance of AF amount. The occurrence of FH non-associated with oligohydramnios is suitable with the explanations proposed by Liu \& Hutson (2000), since it may be the indirect result of passage of urine to the amniotic cavity (19).

In relation to bladder development, the occurrence of $84 \%$ of bladder hypoplasia observed in the present study could be explained both by the lack of filling due to fistula between ureter and urethra or urogenital sinus and by the presence of blind-ending ureter $(11,19)$.
Liu et al. (1999) studied bladder development, using adriamycin and verified that the 7 th gestational day is the critical time when the drug administration causes bladder agenesis. Bladder hypoplasia observed in the present study, considering that the drug was administered on the 8th and 9th days, must be consequent to ureteral defects or represent an effectively distinct anomaly from that observed by the same author (20).

The histomorphometric study revealed increase in the ureteropelvic junction (UPJ) diameter and in pelvic area, and decrease of parenchymal thickness and parenchymal area in the hydronephrosis group when compared with the control group. The parenchyma/pelvis relationships were decreased to half in the hydronephrosis group in relation to the control group, when we used linear measures decreased to one third, when area measurements were used. These results objectively confirm the pelvic dilation and decrease in renal parenchyma observed in kidneys with hydronephrosis.

In models of surgically created hydronephrosis, we detected epithelial, epithelial-mesenchymal, obstructive and inflammatory changes, including atrophy, dysplasia, fibrosis and changes in the renal development $(3,13,14)$.

Microscopic findings in the present study are milder than the ones reported in those works, being restricted to the mechanical repercussion of urine retention, which is characterized by dilation of ureter, pelvis and ducts, and to secondary mass changes, such as atrophy of cortex and medulla. Moreover, no inflammatory or developmental changes were observed.

Other changes were expected in addition to dilation, since urine retention can be associated with renal damage and impair its normal differentiation $(6,14)$. However, the microscopic findings are in agreement with other findings relative to $\mathrm{AFw}$, which indirectly revealed production of fetal urine.

\section{CONCLUSION}

The experimental model of induction by adriamycin showed to be feasible for the microscopic study of fetal hydronephrosis. The microscopic 
changes, despite being milder than the lesions described for surgically produced fetal hydronephrosis, were in agreement with amniotic fluid weight, since they enabled the production of urine with maintenance of the amniotic fluid.

$$
\begin{array}{r}
\overline{\text { Anderson Gonçalves received scholarship for }} \\
\text { Scientific Initiation from CNPq, and Lourenço Sbragia } \\
\text { Neto from FAPESP }
\end{array}
$$

\section{REFERENCES}

1. Brace RA: Physiology of amniotic fluid volume regulation. Clin Obstet Gynecol. 1997; 40: 280-9.

2. Harrison MR, Nakayama DK, Noall R, de Lorimier AA: Correction of congenital hydronephrosis in utero II. Decompression reverses the effects of obstruction on the fetal lung and urinary tract. J Pediatr Surg. 1982; 17: 965-74.

3. Chevalier RL, Thornhill BA, Chang AY: Unilateral ureteral obstruction in neonatal rats leads to renal insufficiency in adulthood. Kidney Int. 2000; 58: 1987-95.

4. Seseke F, Thelen P, Hemmerlein B, Kliese D, Zoller G, Ringert RH: Histologic and molecular evidence of obstructive uropathy in rats with hereditary congenital hydronephrosis. Urol Res. 2000; 28: 104-9.

5. Freedman AL, Bukowski TP, Smith CA, Evans MI, Johnson MP, Gonzalez R: Fetal therapy for obstructive uropathy: diagnosis specific outcomes [corrected]. J Urol. 1996; 156 (2 Pt 2): 720-3; discussion 723-4; Erratum in: J Urol. 1996; 156: 1786.

6. Bernstein J, Risdon RA, Gilbert-Barness E: Renal System. In: Gilbert-Barness E (ed.), Potter's, Pathology of the Fetus and Infant. St Louis, Mosby. 1997; pp. 863-903.

7. Bastide A, Manning F, Harman C, Lange I, Morrison I: Ultrasound evaluation of amniotic fluid: outcome of pregnancies with severe oligohydramnios. Am J Obstet Gynecol. 1986; 154: 895-900.

8. Reddy PP, Mandell J: Prenatal diagnosis. Therapeutic implications. Urol Clin North Amer. 1998; 25: 171-80.

9. Beasley SW, Diez Pardo J, Qi BQ, Tovar JA, Xia HM: The contribution of the adriamycin-induced rat model of the VATER association to our understanding of congenital abnormalities and their embryogenesis. Pediatr Surg Int. 2000; 16: 465-72.

10. Merei J, Hasthorpe S, Farmer P, Hutson JM: Visceral anomalies in prenatally adriamycin-exposed rat fetuses: a model for the VATER association. Pediatr Surg Int. 1999; 15: 11-6.

11. Merei J, Batiha A, Hani IB, El-Qudah M: Renal anomalies in the VATER animal model. J Pediatr Surg. 2001; 36: 1693-7.

12. Franca WM, Goncalves A, Moraes SG, Pereira LA, Sbragia L: Esophageal atresia and other visceral anomalies in a modified Adriamycin rat model and their correlations with amniotic fluid volume variations. Pediatr Surg Int. 2004; 20: 602-8.

13. Steinhardt GF, Liapis H, Phillips B, Vogler G, Nag M, Yoon KW: Insulin-like growth factor improves renal architecture of fetal kidneys with complete ureteral obstruction. J Urol. 1995; 154: 690-3.

14. Steinhardt GF, Salinas-Madrigal L, deMello D, Farber R, Phillips B, Vogler G: Experimental ureteral obstruction in the fetal Opossum: histologic assessment. J Urol. 1994; 152: 2133-8.

15. Tewey KM, Rowe TC, Yang L, Halligan BD, Liu LF: Adriamycin-induced DNA damage mediated by mammalian DNA topoisomerase II. Science. 1984; 226 (4673): 466-8.

16. Orford JE, Cass DT: Dose response relationship between adriamycin and birth defects in a rat model of VATER association. J Pediatr Surg. 1999; 34: 392-8.

17. Beasley SW, Diez Pardo J, Qi BQ, Tovar JA, Xia HM: The contribution of the adriamycin-induced rat model of the VATER association to our understanding of congenital abnormalities and their embryogenesis. Pediatr Surg Int. 2000; 16: 465-72.

18. Kimble RM, Harding JE, Kolbe A: Does gut atresia cause polyhydramnios? Pediatr Surg Int. 1998; 13: 115-7.

19. Liu MI, Hutson JM: Cloacal and urogenital malformations in adriamycin-exposed rat fetuses. BJU Int. 2000; 86: 107-12.

20. Liu MI, Hutson JM, Zhou B: Critical timing of bladder embryogenesis in an adriamycin-exposed rat fetal model: a clue to the origin of the bladder. J Pediatr Surg. 1999; 34: 1647-51.

Received: August 23, 2004

Accepted after revision: November 17, 2004

\begin{tabular}{l}
\hline Correspondence address: \\
Dr. Lourenço Sbragia Neto \\
Discipline of Pediatric Surgery, FCM, Unicamp \\
Rua Alexander Fleming, 181, Barão Geraldo \\
Campinas, SP, 13084-970, Brazil \\
Fax: + 55 19 3788-9473 \\
E-mail: sbragia@ @fm.unicamp.br
\end{tabular}

